\title{
Intravesical Non-Alkalinized Lidocaine Instillation for Interstitial Cystitis/Bladder Pain Syndrome Patients
}

\author{
Teiichiro Aoyagi", Masaaki Tachibana \\ Tokyo Medical University, Ibaraki Medical Center, Inashiki, Japan \\ Email: *aoyagite@tokyo-med.ac.jp
}

Received August 14, 2012; revised September 26, 2012; accepted October 11, 2012

\begin{abstract}
Purpose: The effectiveness of daily or weekly instillation of non-alkalinized lidocaine for patients suffering interstitial cystitis and/or bladder pain syndrome was evaluated retrospectively. Patients and Methods: Five female patients (40 71 years old) diagnosed as interstitial cystitis by cystoscopic findings and a 68-year-old bladder pain syndrome patient were enrolled. All patients, having interstitial cystitis, had undergone hydrodistention therapy previously and had not improved their symptoms by empirical therapies. Daily or weekly (upon their severity of symptoms) intravesical instillation of $20 \mathrm{ml}$ of $4 \%$ non-alkalinized (pH 6.0 - 7.0) lidocaine solution was performed for several times, and patients were asked to keep them in the bladder as long as two hours each time. Previous medications such as anti-cholinergic drugs and analgesics were continued according to patient's requirements and symptoms. The treatment effect was evaluated comparing O'Leary-Sant Symptom Index for interstitial cystitis patients and visual analog pain scale before and after the series of lidocaine therapies. Results: Instillation was made 6 to 16 times. Patients with interstitial cystitis improved their symptoms from O'Leary-Sant Symptom Index 17.5 to 10, Problem Index from 14.8 to 6 in an average. Crouching pain disappeared in all these patients after the instillation therapy. Severe interstitial cystitis findings on cystoscopy disappeared completely in one patient after the therapy. One patient having bladder pain syndrome reduced her analgesics use, and bladder-filling pain decreased from 7 to 3 as a visual analog scale score. One patient complained palpitation at 11th instillation and abandoned treatment thereafter, otherwise, none of these patients showed side effect concerning lidocaine toxicity. Conclusions: Intravesical non-alkalinized lidocaine instillation therapy for interstitial cystitis/bladder pain syndrome patients were an easy, safe and effective treatment.
\end{abstract}

Keywords: Bladder Pain Syndrome; Interstitial Cystitis; Lidocaine; Instillation Therapy

\section{Introduction}

Interstitial cystitis/bladder pain syndrome (IC/BPS) is the clinical diagnosis for distressing symptoms characterized by pelvic pain and urinary symptoms in the form of urgency, frequency and nocturia. Clear definition of IC is made by cystoscopic findings known as Hunner's ulcer, however the National Institute of Diabetes and Digestive and Kidney Diseases (NIDDK) defined more clinical and strict criteria which is said to exclude about $60 \%$ of expected cases [1]. The name BPS is used to include more majority cases, whose contrary may consist with more variable pathological condition with similar symptoms [2]. Therefore over 180 therapies have been tried for the treatment to these symptoms without standardized effectiveness [3]. Among these treatments, intravesical therapies were tried with enthusiasm because the pathogenesis of these symptoms presumed to be in the bladder wall [4,5]. American Urological Association (AUA) published

\footnotetext{
"Corresponding author.
}

the guideline for diagnosis and treatment of IC/BPS in 2011 [6]. According to this guideline, intravesical drug instillation therapies are listed as second line treatment, and relief of pain is advocated one of the most important treatment purpose. Among the intravesical instillation materials, lidocaine had been used expecting its anesthetic effect to control severe bladder pain. We tried intravesical instillation of lidocaine solution, which was conventionally used for male urethral anesthesia before cystoscopy, for incurable bladder pain patients, who had not respond any medication nor hydrodistention therapy. And we here report the effectiveness of intravesical nonalkalinized lidocaine instillation therapy for six IC/BPS patients.

\section{Patients and Methods}

Among the patients visited from 2009 to 2010, and could be followed more than one year, five female patients (40 71 years old) diagnosed as interstitial cystitis by cystoscopic 
Table 1. Patient record.

\begin{tabular}{ccccccccc}
\hline & Age & Disease & $\begin{array}{c}\text { Duration of } \\
\text { disease }\end{array}$ & $\begin{array}{c}\text { Instillation frequency } \\
\text { (times) }\end{array}$ & Symptom score & Problem score & Duration of effect & Adverse events \\
\hline 1 & 50 & IC & $4 \mathrm{Y}$ & $1 / \mathrm{w} \times 6$ & $13 \rightarrow 11$ & $15 \rightarrow 8$ & $6 \mathrm{M}$ & None \\
2 & 61 & IC & $3 \mathrm{M}$ & $1 /$ day $\times 10$ & NA & NA & $2.5 \mathrm{Y}$ & None \\
3 & 67 & IC & $15 \mathrm{Y}$ & $1 / \mathrm{w} \times 6$ & $18 \rightarrow 12$ & $15 \rightarrow 4$ & $2 \mathrm{Y}$ & None \\
4 & 71 & IC & $1 \mathrm{Y}$ & $1 / \mathrm{w} \times 16$ & $20 \rightarrow 15$ & $10 \rightarrow 3$ & $16 \mathrm{M}$ & None \\
5 & 40 & IC & $4 \mathrm{Y}$ & $1 / \mathrm{w} \times 6$ & $19 \rightarrow 2$ & $16 \rightarrow 2$ & $7 \mathrm{Y}$ & None \\
6 & 68 & PBS & $1 \mathrm{Y}$ & $1 / 2 \mathrm{w} \times 11$ & VAS & $7 \rightarrow 3$ & & Palpitation \\
\hline
\end{tabular}

IC: Interstitial cystitis; Y: Year; M: Month; w: Week; PBS: Painful bladder syndrome.

findings and a 68-year-old bladder pain syndrome patient were enrolled. All patients, having interstitial cystitis, had undergone hydrodistention therapy previously and had not improved their symptoms by empirical therapies, such as medication of supratast tosilate, anti-cholinergics and analgesics. Daily or weekly (according to their severity of symptoms) intravesical instillation of $20 \mathrm{ml}$ of $4 \%$ non-alkalinized ( $\mathrm{pH} 6.0$ - 7.0) lidocaine solution (Astra Zeneca) was performed for several times, and patients were asked to keep them in the bladder as long as two hours each time. Previous medications were continued according to patient's requirements and symptoms. The treatment effect was evaluated comparing O'Leary-Sant Symptom Index for interstitial cystitis patients (Japanese translated version) [7] and visual analog pain scale before and after the series of lidocaine therapies respectively. Instillation therapy continued until each patient felt the improvement of severe symptoms enough to quit the continuous therapy, or experience an adverse event suggesting any relation to this therapy.

\section{Results}

Profiles of patients and treatment results are summarized in the Table 1. Instillation was made 6 to 16 times. Patients with interstitial cystitis improved their symptoms in O'Leary-Sant Symptom Index from 17.5 to 10.0, in Problem Index from 14.8 to 6.0 in an average. Crouching pain (one can not keep standing due to severe bladder pain) disappeared in all these patients after the instillation therapy. Severe inflammatory cystoscopic findings disappeared completely in one IC patient after the therapy as shown in the Figure 1. One patient having bladder pain syndrome reduced her analgesics use, and bladder filling pain decreased from 7 to 3 as a visual analog scale score. This BPS patient complained palpitation after an 11th instillation and abandoned treatment thereafter, otherwise, none of these patients showed side effect concerning lidocaine toxicity. The treatment effect lasted three months to two years. All these patients re-started

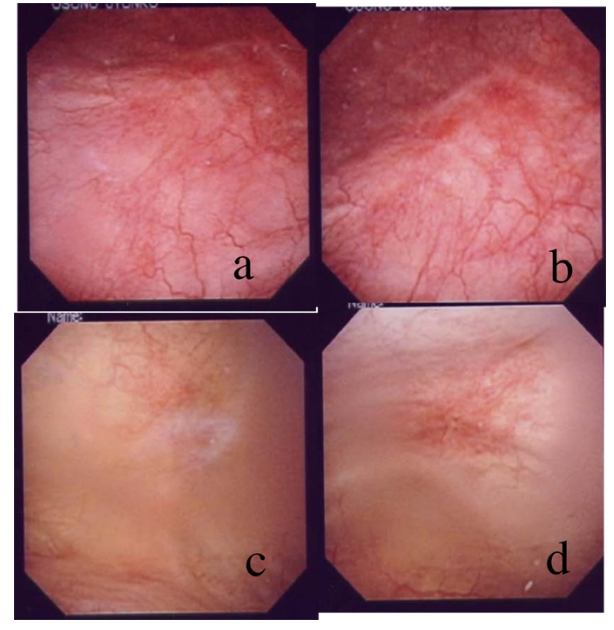

Figure 1. Cystoscopic findings before $(a, b)$ and after $(c, d)$ the lidocaine therapy in Patient \#5.

lidocaine instillation therapy when their symptoms recurred, and severe symptoms were re-stabilized after two to three weekly instillations.

\section{Discussion}

IC/BPS is a symptomatic and clinical diagnostic disease, which had never been defined pathologically [2]. Consequently its management was mainly empirical and symptomatic, and has no standard therapy though some management guidelines have been published in several countries [2,8]. Pentosan polysulphate [9] and heparin [10] were used to augment altered surface glycosaminoglycan of bladder mucosa, which is presumed to make dysfunction of mucosa permeability and cause bladder pain. Amitriptyline, hydroxyzine and cimetidine have been used to block histamine from mast cell that was presumed to be recruited by autoimmune inflammatory reaction [3]. Suplatast tosilate and steroids have been used to reduce inflammation or allergic reaction itself [11]. Resiniferatoxin was used to de-sensitization of $\mathrm{C}$ nerve fiber for the purpose of reducing severe pain in 
voiding and/or bladder filling [12]. Lidocaine had been used just expecting for its local analgesic effect [13], and several report supported the promising results [14-16].

To increase the mucosal permeability of lidocaine, alkalinization of solution or electro-stimulation were used $[14,17]$. We used non-modified commercialized 4\% lidocaine solution ( $\mathrm{pH} \mathrm{6.0)}$ and instilled $20 \mathrm{ml}$ solution to the bladder in one time. Tissue penetration occurs when the ionized liquid-soluble lidocaine ( $\mathrm{pKa} \pm 8.0)$ is buffered by the surrounding tissue, and the drug converts into the lipid soluble base form [18]. The amount of lidocaine solution might be decreased if bladder mucosa is widely damaged or inflamed by infection, consequently showing more alkaline urine. Pode et al. [13] reported the mean serum lidocaine concentration was $0.16 \pm 0.03$ (SE) $\mu \mathrm{g} / \mathrm{ml}$ by using $750 \mathrm{mg}$ of intravesical non-alkalinized lidocaine instillation to the bladder, which was far below the toxic serum concentration of lidocaine (greater than 5 $\mu \mathrm{g} / \mathrm{ml}$ ). Henry et al. [14] reported that the amount of absorbed lidocaine in IC patients from bladder mucosa was the same as that of healthy volunteers. According to these results we concluded that our one time dose: intravesical $20 \mathrm{ml}$ of $4 \%$ non-alkalinized lidocaine solution, should be basically safe for IC/BPS patients.

We experienced the improvement of not only symptom but also cystoscopic findings in one patient by consecutive instillation of lidocaine. Although the exact mechanism is unclear, it is presumed that relief of pain discontinues the vicious cycle of some allergic reaction followed by inflammation and bladder pain. We asked the patients to keep lidocaine solution in the bladder at least for two hours. Lee et al. reported intravesical device mediated lidocaine instillation, which enables continuous lidocaine release as long as three days [19]. It should be helpful for IC/BPS patients if this device developed for more practical use [20].

We used commercially available 4\% lidocaine solution that can be used for the urethral topical anesthesia for cystoscopic examination, and obtained satisfactory results. We empirically used the same lidocaine instillation for the relief of severe pain induced by BCG instillation therapy. And this time we used it for IC/BPS syndrome patients. Although we did not apply strict scientific methodology such as randomized prospective study in this report, we are convinced that the repot of actual usage and effect of lidocaine will provide some help in the treatment of these patients in clinical practice. We conclude that intravesical non-alkalinized lidocaine instillation therapy is a safe, easy and effective treatment for IC/BPS patients, and we think it should be used more prevalently as a recommended therapy.

\section{REFERENCES}

[1] P. M. Hanno, J. R. Landis, Y. Matthews-Cook, J. Kusek, and L. Nyberg Jr., "The Diagnosis of Interstitial Cystitis Revised; Lessons Learned from the National Institute of Health Interstitial Cystitis Database Study," The Journal of Urology, Vol. 161, No. 2, 1999, pp. 553-557. doi:10.1016/S0022-5347(01)61948-7

[2] J. P. van de Merwe, J. Nordling, P. Bouchelouche, et al., "Diagnostic Criteria, Classification, and Nomenclature for Painful Bladder Syndrome/Interstitial Cystitis: An ESSIC Proposal," European Urology, Vol. 53, No. 1, 2008, pp. 60-67. doi:10.1016/j.eururo.2007.09.019

[3] M. Fall, F. Oberpenning and R. Peeker, "Treatment of Bladder Pain Syndrome/Interstitial Cystitis 2008: Can We Make Evidence-Based Decisions?" European Urology, Vol. 54, No. 1, 2008, pp. 65-78. doi:10.1016/j.eururo.2008.03.086

[4] T. C. Lau and J. M. Bengtson, "Management Strategies for Painful Bladder Syndrome," Reviews in Obstetrics \& Gynecology, Vol. 3, No. 2, 2010, pp. 42-48.

[5] R. Taneja, "Intravesical Lignocaine in the Diagnosis of Bladder Pain Syndrome," International Urogynecology Journal, Vol. 21, No. 3, 2010, pp. 321-324. doi:10.1007/s00192-009-1045-0

[6] P. M. Hanno, D. A. Burks, J. Q. Clemens, et al., "Diagnosis and Treatment of Interstitial Cystitis/Bladder Pain Syndrome," 2011.

http://www.auanet.org/content/clinical-practice-guideline s/clinical-guidelines/main-reports/ic-bps/diagnosis_and_tr eatment_ic-bps.pdf

[7] M. P. O’Leary, G. R. Sant, F. J. Fowler Jr., K. E. Whitmore and J. Spolarich-Kroll, "The Interstitial Cystitis Symptom Index and Problem Index," Urology, Vol. 49, No. 5A, 1997, pp. 58-63. doi:10.1016/S0090-4295(99)80333-1

[8] J. Nordling, M. Fall and P. M. Hanno, "Global Concepts of Bladder Pain Syndrome (Interstitial Cystitis)," World Journal of Urology, Vol. 30, No. 4, 2012, pp. 457-464. doi:10.1007/s00345-011-0785-X

[9] G. Chiang, P. Patra, R. Letourneau, et al., "Pentosanpolysulfate Inhibits Mast Cell Histamine Secretion and Intracellular Calciumion Levels: An Alternative Explanation of Its Beneficial Effect in Interstitial Cystitis," The Journal of Urology, Vol. 164, No. 6, 2000, pp. 2119-2125. doi:10.1016/S0022-5347(05)66981-9

[10] C. L. Parsons, T. Housley, J. D. Schmidt and D. Lebow, "Treatment of Interstitial Cystitis with Intravesical Heparin," British Journal of Urology, Vol. 73, No. 5, 1994, pp. 504-507. doi:10.1111/j.1464-410X.1994.tb07634.x

[11] T. Ueda, M. Tamaki, O. Ogawa, T. Yamauchi and N. Yoshimura, "Improvement of Interstitial Cystitis Symptoms and Problems That Developed during Treatment with Oral IPD-1151T," The Journal of Urology, Vol. 164, No. 6, 2000, pp. 1917-1920. doi:10.1016/S0022-5347(05)66917-0

[12] M. Lazzeri, M. Spinelli, P. Beneforti, et al., "Intravesical Infusion of Resiniferatoxin by a Temporary in Situ Drug Delivery System to Treat Interstitial Cystitis: A Pilot Study," European Urology, Vol. 45, No. 1, 2004, pp. 98-102. doi.org/10.1016/S0302-2838(03)00418-4

[13] D. Pode, E. Zylber-Kats and A. Shapiro, "Intravesical 
Lidocaine: Topical Anesthesia for Bladder Mucosal Biopsies," The Journal of Urology, Vol. 148, No. 3, 1992, pp. 795-796.

[14] R. Henry, L. Patterson, N. Avery, et al., "Absorption of Alkalinized Intravesical Lidocaine in Normal and Inflamed Bladders: A Simple Method for Improving Bladder Anesthesia," The Journal of Urology, Vol. 165, No. 6, 2001, pp. 1900-1903.

[15] J. C. Nickel, R. Moldwin, S. Lee, E. L. Davis, R. A. Henry and M. G. Wyllie, "Intravesical Alkalinized Lidocaine (PDS597) Offers Sustained Relief from Symptoms of Interstitial Cystitis and Painful Bladder Syndrome," BJU International, Vol. 103, No. 7, 2009, pp. 910-918. doi:10.1111/j.1464-410X.2008.08162.x

[16] B. K. Welk and J. M. H. Teichman, "Dyspareunia Response in Patients with Interstitial Cystitis Treated with Intravesical Lidocaine, Bicarbonate, and Heparin," Urology, Vol. 71, No. 1, 2008, pp. 67-70. doi:10.1016/j.urology.2007.09.067

[17] T. Gurpinar, H. Y. Wong and D. P. Griffith, "Electromo- tive Administration of Intravesical Lidocaine in Patients with Interstitial Cystitis," Journal of Endourology, Vol. 10, No. 5, 1996, pp. 443-447. doi:10.1089/end.1996.10.443

[18] G. T. Tucker and L. E. Mather, "Properties, Absorption, and Disposition of Local Anesthetic Agents," In: M. J. Cousins, P. O. Bridenbaugh and E. D. Neural, Eds., Blockage in Clinical Anesthesia and Management of Pain, Lippincott-Raven, Philadelphia, 1998, pp. 55-86.

[19] H. Lee and M. J. Cima, "An Intravesical Device for the Sustained Delivery of the Bladder," Journal of Controlled Release, Vol. 149, No. 2, 2011, pp. 133-139. doi:10.1016/j.jconrel.2010.10.016

[20] J. C. Nickel, P. Jain, N. Shore, et al., "Continuous Intravesical Lidocaine Treatment for Interstitial Cystitis/Bladder Pain Syndrome: Safety and Efficacy of a New Drug Delivery Device," Science Translational Medicine, Vol. 4, No. 143, 2012, p. 143ra100. doi:10.1126/scitranslmed.3003804 